\title{
The impact of blood pressure management after spinal cord injury: a systematic review of the literature
}

\author{
Yamaan S. Saadeh, MD, Brandon W. Smith, MD, Jacob R. Joseph, MD, Sohaib Y. Jaffer, BA, \\ Martin J. Buckingham, MD, Mark E. Oppenlander, MD, Nicholas J. Szerlip, MD, and Paul Park, MD \\ Department of Neurosurgery, University of Michigan, Ann Arbor, Michigan
}

OBJECTIVE Spinal cord injury (SCI) results in significant morbidity and mortality. Improving neurological recovery by reducing secondary injury is a major principle in the management of $\mathrm{SCl}$. To minimize secondary injury, blood pressure (BP) augmentation has been advocated. The objective of this study was to review the evidence behind BP management after SCl.

METHODS This systematic review was conducted following the PRISMA (Preferred Reporting Items for Systematic Reviews and Meta-Analyses) guidelines. Using the PubMed database, the authors identified studies that investigated BP management after acute SCI. Information on BP goals, duration of BP management, vasopressor selection, and neurological outcomes were analyzed.

RESULTS Eleven studies that met inclusion criteria were identified. Nine studies were retrospective, and 2 were singlecohort prospective investigations. Of the 9 retrospective studies, 7 reported a goal mean arterial pressure (MAP) of higher than $85 \mathrm{~mm} \mathrm{Hg}$. For the 2 prospective studies, the MAP goals were higher than $85 \mathrm{~mm} \mathrm{Hg}$ and higher than 90 $\mathrm{mm} \mathrm{Hg}$. The duration of BP management varied from more than 24 hours to 7 days in 6 of the retrospective studies that reported the duration of treatment. In both prospective studies, the duration of treatment was 7 days. In the 2 prospective studies, neurological outcomes were stable to improved with BP management. The retrospective studies, however, were contradictory with regard to the correlation of BP management and outcomes. Dopamine, norepinephrine, and phenylephrine were the agents that were frequently used to augment BP. However, more complications have been associated with dopamine use than with the other vasopressors.

CONCLUSIONS There are no high-quality data regarding optimal BP goals and duration in the management of acute $\mathrm{SCl}$. Based on the highest level of evidence available from the 2 prospective studies, MAP goals of $85-90 \mathrm{~mm} \mathrm{Hg} \mathrm{for} \mathrm{a}$ duration of 5-7 days should be considered. Norepinephrine for cervical and upper thoracic injuries and phenylephrine or norepinephrine for mid- to lower thoracic injuries should be considered.

https://thejns.org/doi/abs/10.3171/2017.8.FOCUS17428

KEY WORDS spinal cord injury; blood pressure; MAP goals; MAP duration; vasopressors; mean arterial blood pressure

$\mathrm{S}$ PINAL cord injury (SCI) is estimated to occur at an incidence approximating 17,000 cases per year, and there are currently approximately 300,000 people living with the sequelae of a SCI. Spinal cord injury results in significant personal costs and considerably decreases independence as well as quality of life. Lifetime expenses resulting from a SCI range from $\$ 1.1$ million to $\$ 2.6$ million, depending on extent of injury. ${ }^{23}$ Despite significant research investment, there has been modest advancement in the perioperative and postoperative management of patients with SCI.

The principles of management for SCI generally include

ABBREVIATIONS AANS = American Association of Neurological Surgeons; AIS = ASIA Impairment Scale; ASIA = American Spinal Injury Association; BP = blood pressure; CNS = Congress of Neurological Surgeons; $\mathrm{MAP}=$ mean arterial pressure; $\mathrm{SCl}=$ spinal cord injury .

SUBMITTED July 1, 2017. ACCEPTED August 7, 2017.

INCLUDE WHEN CITING DOI: 10.3171/2017.8.FOCUS17428. 
early decompression and stabilization, ${ }^{5}$ with prevention of secondary injury following the initial insult. A number of strategies have been investigated for minimizing secondary injury, including blood pressure (BP) augmentation with volume support and vasopressors; therapeutic hypothermia: ${ }^{15}$ medications to decrease inflammation, such as riluzole, ${ }^{3}$ glibenclamide, ${ }^{28}$ and Rho-antagonists, ${ }^{2}$ and steroids, as well as agents promoting plasticity and axonal regeneration, such as Nogo-A. ${ }^{1}$ However, many of these interventions remain at the trial stage and have not advanced to routine clinical use.

$\mathrm{BP}$ control in the management of SCI remains an area of frequent discussion, although there are limited highquality data and the strength of management recommendations is limited. Current recommendations according to the guidelines of the American Association of Neurological Surgeons/Congress of Neurological Surgeons (AANS/ CNS) Joint Section on Spine and Peripheral Nerves advise correcting hypotension and maintaining a mean arterial pressure (MAP) goal of 85-90 $\mathrm{mm} \mathrm{Hg}$ for 7 days postinjury. ${ }^{32}$ The Consortium for Spinal Cord Medicine formally recommends avoiding hypotension. ${ }^{10}$

There is general agreement as well as evidence that hypotension with neurological injury results in poorer outcomes $;, 8,25$ however, evidence that increasing BP promotes improved outcomes is significantly more limited. In addition, it is not clear if the costs of promoting higher BP for a longer duration of time, which include invasive monitoring, risks of vasopressor use, and longer intensive care unit stays, outweigh the benefits.

The goal of this systematic review was to compile and analyze the data on BP management in patients who have sustained SCI and to examine the current recommendations and the basis on which they are made. Specifically, this paper focuses not only on BP goals and duration of $\mathrm{BP}$ management but also examines the choice of vasopressors.

\section{Methods}

\section{Literature Search and Inclusion Criteria}

We conducted a systematic review according to PRISMA (Preferred Reporting Items for Systematic Reviews and Meta-Analyses) guidelines. ${ }^{22}$ Using the PubMed database, we first identified relevant articles using the search terms "spinal cord injury" AND "blood pressure" published until July 25, 2017. Based on review of the article titles, we selected relevant titles related to our review. We excluded articles that were not written in the English language. These titles underwent an abstract review, after which unrelated titles were excluded. Additional relevant publications were identified and added after review of reference lists. The remaining articles underwent a full-text review. Articles without full text were excluded. Animal studies were also excluded.

\section{Data Extraction and Outcome Measures}

We extracted the following data from each relevant article: first author, year of publication, study type, presence of comparison group, length of follow-up, MAP goal, MAP goal duration, and outcome measures.

\section{Results \\ Study Selection}

We identified a total of 1743 articles through our database search. After title review and removal of duplicates, 1689 articles were excluded, and 56 articles underwent abstract and full-text review. After reference review, 4 additional studies were included. Forty-five articles were excluded on full-text review. The reasons for exclusion of studies were incorrect study type, foreign language, animal studies, inability to obtain the full text, and lack of collection of data on neurological outcome. Eleven studies met the criteria for review. Two of these studies were prospective and 9 were retrospective. The search flow diagram is displayed in Fig. 1.

\section{Relationship of MAP Goals to Outcomes}

A total of 11 studies were identified in which BP and neurological outcome data for SCI patients during the acute postinjury period were collected (Table 1). Two studies were prospective. These studies evaluated the outcomes of patients who suffered SCIs and were managed after injury and stabilization following protocols that included targeted MAP goals. ${ }^{20,31}$ Nine studies were retrospective reviews that collected information on MAP after SCI as well as neurological outcome measures throughout the postinjury period..$^{6,9,16,17,19,21,27,33}$

Levi et al. ${ }^{20}$ performed a prospective study in which the authors described the outcomes of a group of $50 \mathrm{pa}-$ tients who underwent spinal immobilization or fixation as indicated, with their postinjury care at a trauma center between 1990 and 1991. An MAP goal higher than 90 $\mathrm{mm} \mathrm{Hg}$ was maintained with fluids and dopamine for the 1st week after injury, although some patients required the addition of dobutamine for additional support. Given that $82 \%$ of patients showed stable or improved neurological function at the 6-week follow-up as measured by their Frankel grade, the authors concluded that pursuing aggressive MAP goals was feasible and of relatively low risk. There was no control group.

Vale et al. ${ }^{31}$ performed a prospective study describing the results of 77 patients with SCI who were treated at their institution between 1992 and 1997. These patients underwent spinal immobilization or fixation. Their postoperative care included an MAP goal higher than $85 \mathrm{~mm} \mathrm{Hg}$ for 7 days. Sixty-four patients underwent follow-up for at least 12 months postinjury, and all patients showed stable examination or neurological improvement at the 12-month follow-up as measured by the American Spinal Injury Association (ASIA) motor index score and the ASIA Impairment Scale (AIS) grade. There was no comparison group. The authors concluded that BP augmentation improves neurological outcomes but stated the need for randomized trials to identify the ideal MAP goal.

Wolf et al. ${ }^{33}$ retrospectively reviewed data for 52 patients who sustained a SCI between 1987 and 1990 due to bilateral facet dislocation. The patients were managed after decompression with an MAP goal greater than $85 \mathrm{~mm}$ $\mathrm{Hg}$ for 5 days. Neurological function was recorded using the modified Frankel grade of Benzel and Larson ${ }^{4}$ as well as the Yale Scale score. Twenty-two patients underwent 


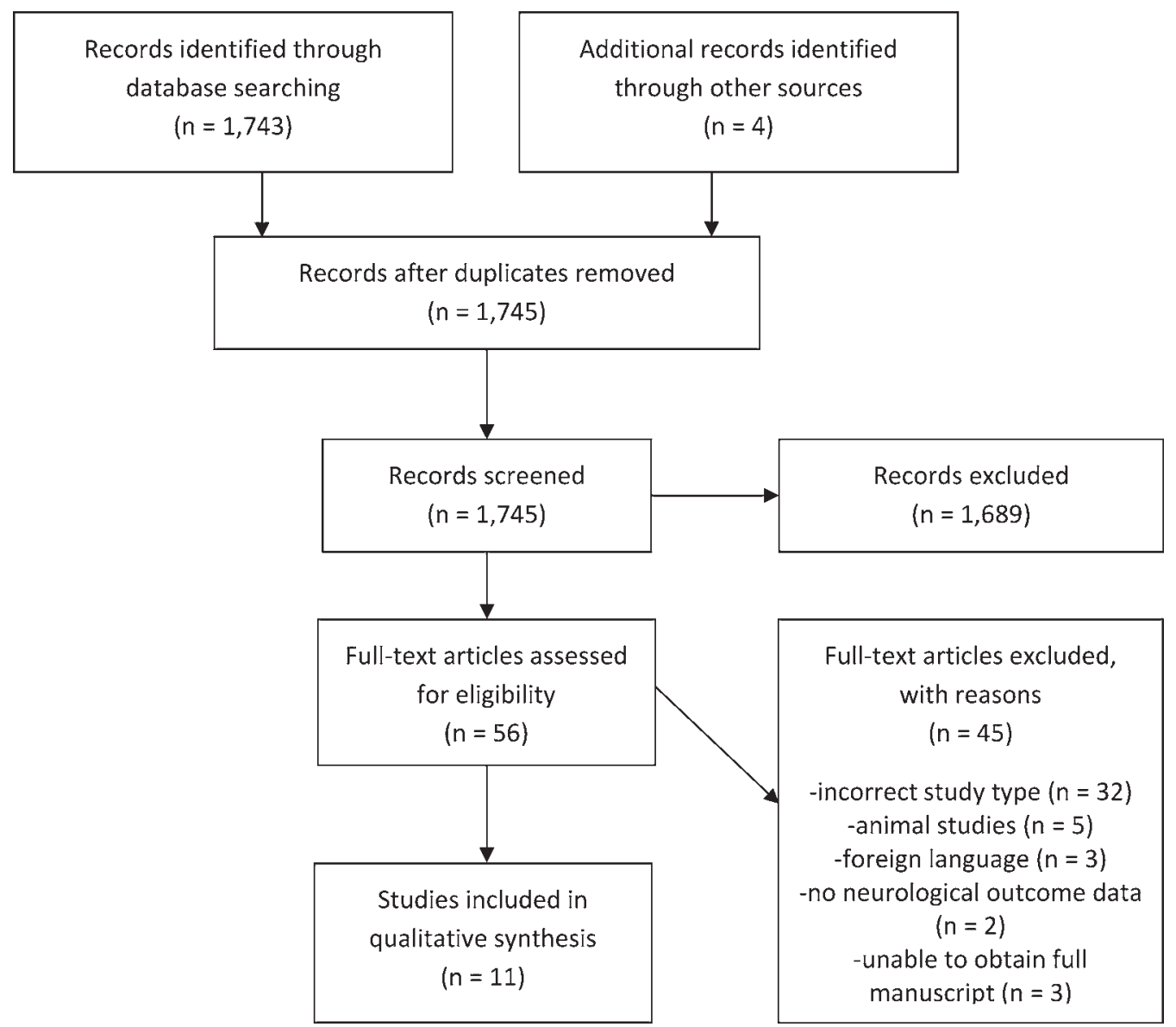

FIG. 1. Flow diagram showing the selection process for this systematic review of $\mathrm{BP}$ management after $\mathrm{SCl}$.

follow-up for at least 12 months postinjury. All of these patients had stability or improvement in their functional grade. There were no comparison or control groups.

Cohn et al. ${ }^{7}$ retrospectively reviewed 17 patients presenting with quadriplegia between 2000 and 2006 in whom MAP recording was performed at least 3 times daily for 7 days postinjury. The authors estimated the amount of time patients spent with MAP above thresholds of 85 , 75 , and $65 \mathrm{~mm} \mathrm{Hg}$. The authors estimated that patients had MAP greater than $85 \mathrm{~mm} \mathrm{Hg} \mathrm{33 \%}$ of the time, greater than $75 \mathrm{~mm} \mathrm{Hg} 65 \%$ of the time, and greater than $65 \mathrm{~mm}$ $\mathrm{Hg} 91 \%$ of the time. Neurological outcome as measured by AIS grade and ASIA motor score was not found to be related to duration of time at a goal of MAP greater than 75 or $85 \mathrm{~mm} \mathrm{Hg}$. Patients underwent follow-up until the time of discharge.

Hawryluk et al. ${ }^{16}$ retrospectively reviewed MAP data for 74 SCI patients who underwent postinjury treatment between 2005 and 2011 and were managed with an MAP goal greater than $85 \mathrm{~mm} \mathrm{Hg}$ for 5 days postinjury. This patient population is a subset of the group that was initially studied in Inoue et al., ${ }^{17}$ the results of which are described below. Although this was a retrospective review, the MAP data were collected prospectively. The authors collected 1-minute-interval MAP measurements and interestingly found that about $25 \%$ of all MAPs for the first 5 days postinjury were lower than the goal. The patients who exhibited the greatest neurological improvement as measured by AIS grade had fewer MAP measurements lower than the goal compared with patients without neurological improvement. The authors reported that their data suggested that an MAP of 70-75 $\mathrm{mm} \mathrm{Hg}$ appeared to be the threshold at which neurological benefit is correlated with MAP goals. In addition, the authors noted that the first 2-3 days after injury with elevated MAP correlated most strongly with recovery. Patients underwent follow-up until the time of discharge.

Readdy et al. ${ }^{27}$ retrospectively analyzed 34 patients who presented with traumatic central cord syndrome between 2005 and 2011 and had MAP goals of higher than $85 \mathrm{~mm} \mathrm{Hg}$ for greater than 24 hours. AIS grades were obtained as a measure of neurological outcome, and patients underwent follow-up throughout their hospitalization until discharge. Fifty-six percent of patients improved at least 1 neurological grade by the time of discharge. The remaining patients had an unchanged AIS grade at discharge.

Inoue et al. ${ }^{17}$ retrospectively reviewed 131 patients who were admitted with SCI between 2005 and 2011 and received vasopressors to maintain MAP goals of higher than $85 \mathrm{~mm} \mathrm{Hg}$. Although this was a retrospective review, the MAP data were collected prospectively. This patient population was also analyzed in the studies by Hawryluk et al. ${ }^{16}$ and Catapano et al., ${ }^{6}$ which are also reviewed in this paper. MAP goals were maintained for 5 days before 
TABLE 1. Pertinent characteristics of the 11 articles included in this systematic review of BP management after spinal cord injury

\begin{tabular}{|c|c|c|c|c|c|c|c|c|}
\hline Authors \& Year & $\begin{array}{c}\text { Data } \\
\text { Collection }\end{array}$ & $\begin{array}{l}\text { Study } \\
\text { Type }\end{array}$ & $\begin{array}{l}\text { Comparison } \\
\text { Group }\end{array}$ & $\begin{array}{c}\text { Neurological Outcome } \\
\text { Measure }\end{array}$ & $\begin{array}{l}\text { No. of } \\
\text { Pts }\end{array}$ & $\begin{array}{l}\text { MAP Goal } \\
(\mathrm{mm} \mathrm{Hg})\end{array}$ & $\begin{array}{l}\text { MAP Goal } \\
\text { Duration }\end{array}$ & $\begin{array}{l}\text { Length of } \\
\text { Follow-Up }\end{array}$ \\
\hline Catapano et al., 2016 & Retrospective & Case series & No & AIS grade & 62 & NS & NS & Until discharge \\
\hline Cohn et al., 2010 & Retrospective & Case series & No & $\begin{array}{l}\text { AIS grade, ASIA motor } \\
\text { score }\end{array}$ & 17 & NS & NS & Until discharge \\
\hline Dakson et al., 2017 & Retrospective & Comparative & Yes & $\begin{array}{l}\text { AIS grade, ASIA motor } \\
\text { score }\end{array}$ & $94^{*}$ & $>85$ & 5 days & $\begin{array}{l}\text { After neurorehabili- } \\
\text { tation }\end{array}$ \\
\hline Hawryluk et al., 2015 & Retrospective & Case series & No & AIS grade & 74 & $>85$ & 5 days & Until discharge \\
\hline Inoue et al., 2014 & Retrospective & Case series & No & AIS grade & 131 & $>85$ & 7 days & Until discharge \\
\hline Kepler et al., 2015 & Retrospective & Case series & No & ASIA motor score & 92 & $>85$ & $\geq 5$ days & Until hospital Day 5 \\
\hline Levi et al., 1993 & Prospective & Case series & No & Frankel grade & 50 & $>90$ & 7 days & 6 wks \\
\hline Martin et al., 2015 & Retrospective & Case series & No & ASIA motor score & 105 & NS & NS & Until discharge \\
\hline Readdy et al., 2015 & Retrospective & Case series & No & AIS grade & 34 & $>85$ & $>24 \mathrm{hrs}$ & Until discharge \\
\hline Vale et al., 1997 & Prospective & Case series & No & $\begin{array}{l}\text { AIS grade, ASIA motor } \\
\text { score }\end{array}$ & 77 & $>85$ & 7 days & $12 \mathrm{mos}$ \\
\hline Wolf et al., 1991 & Retrospective & Case series & No & $\begin{array}{l}\text { Modified Frankel score, } \\
\text { Yale Scale score }\end{array}$ & 52 & $>85$ & 5 days & $12 \mathrm{mos}$ \\
\hline
\end{tabular}

NS = not specified; Pts = patients.

* Serial MAP data collected for 50 of these patients.

being relaxed to lower levels. AIS grades were collected as outcome measures up until the time of discharge from the hospital; no association was found between neurological outcome and the use of vasopressors to maintain MAP goals. There was no comparison or control group.

Kepler et al. ${ }^{19}$ retrospectively reviewed 92 patients who were admitted between 2006 and 2009 for an acute SCI, with an MAP goal of higher than $85 \mathrm{~mm} \mathrm{Hg}$ for at least 5 days. Outcomes compared the ASIA motor scores on admission and on hospital Day 5, which showed an overall decrease in neurological function over that period of an average of 2.2 points on the ASIA motor impairment scale. There was no comparison or control group.

Martin et al. ${ }^{21}$ retrospectively reviewed acute cervical and thoracic SCIs in 105 patients between 2007 and 2009 and evaluated the average MAP and lowest MAP hourly for the first 3 days after hospitalization. MAP levels were collected for the first 72 hours of the patient's hospitalization period. ASIA motor scores were collected as outcome measures during the hospitalization period. There was no correlation between neurological outcomes and the use of vasopressors or number of failures to meet MAP goals.

Catapano et al. ${ }^{6}$ retrospectively reviewed 62 patients who presented between 2005 and 2011 with traumatic SCI. This patient population was also previously studied by Inoue et al. ${ }^{17}$ as well as by Hawryluk et al. ${ }^{16}$ both of which are summarized above. The authors compared the average MAPs as well as the proportion of MAPs lower than $85 \mathrm{~mm} \mathrm{Hg}$ with their outcomes, as measured by comparing AIS grades at presentation and discharge. MAPs were analyzed only for the first 3 days after injury. There was a correlation between improvement and a greater proportion of MAPs higher than $85 \mathrm{~mm} \mathrm{Hg}$ in patients presenting with AIS Grade A, B, or C.

Dakson et al. ${ }^{11}$ retrospectively reviewed 94 patients who presented with SCI between 2006 and 2010. Serial hourly
MAPs were collected for 50 of these patients. MAP lower than $85 \mathrm{~mm} \mathrm{Hg}$ for more than 2 consecutive hours in the 5 days postinjury was defined as suboptimal BP management. AIS grade and ASIA motor scores were collected as neurological outcome data. Forty-one patients (82\%) did not meet the MAP goal in this study. The 9 patients (18\%) who did meet the MAP goal demonstrated an improved AIS grade compared with patients who did not meet the MAP goal, as well as a statistically significantly greater improvement in ASIA motor score at discharge from acute care. The improvement in AIS grade for the group that achieved MAP goals persisted at later points of follow-up, although the ASIA motor score improvement did not persist.

\section{MAP Goal Duration}

Current recommendations for the duration of elevated MAP goals are 7 days of elevated MAP to $85-90 \mathrm{~mm}$ $\mathrm{Hg}$ per the guidelines of the AANS/CNS Joint Committee on Spine and Peripheral Nerves. ${ }^{32}$ This duration is derived from the protocol of the studies by Levi, Vale, and their colleagues, which were performed in the 1990s. In the Vale ${ }^{31}$ study, 7 days was chosen as the duration based on experimental data on maximum swelling measured at 5 days postinjury in experimentally induced SCI in monkeys. ${ }^{34} \mathrm{~A}$ number of other studies on MAP goals in SCI have had protocols with an MAP goal duration of less than 7 days. It does not appear that there are any studies that compare outcomes related to different durations of MAP goals.

Hawryluk et al.$^{16}$ collected 1-minute-interval MAP measurements for patients who were admitted with SCI and found that average MAP values correlated most strongly with neurological outcome for the first 2-3 days after injury. The authors did find that the proportion of MAP measurements below the BP goal correlated with outcome for 
the first 7 days after injury. Hawryluk,${ }^{16}$ Kepler ${ }^{19}$ Inoue,${ }^{17}$ Wolf, ${ }^{33}$ and Dakson ${ }^{11}$ and their colleagues described methods in which elevated MAP goals were pursued for 5 days postinjury, rather than 7 days, and they did not report the occurrence of an associated neurological decline with the shorter duration of elevated BP goals.

\section{Vasopressor Selection}

Among the studies referenced, there were a variety of different vasopressors used to maintain the respective MAP goals. The most recent AANS/CNS Joint Committee guidelines do not make a specific recommendation regarding the type of vasopressor to use for their recommendation of elevated MAP goals of $85-90 \mathrm{~mm} \mathrm{Hg}{ }^{32}$ The Consortium for Spinal Cord Medicine ${ }^{10}$ recommends dopamine or norepinephrine for cervical or high thoracic regions, as hypotension may result from loss of sympathetic tone and require an agent with alpha and beta adrenergic effect. For low thoracic regions, phenylephrine is suggested, as vasodilation is suspected to be the cause of hypotension.

Inoue et al. ${ }^{17}$ described in detail their experiences with the use of dopamine and phenylephrine. Dopamine was found to cause major complications at a rate of $10 \%$ compared with phenylephrine, which caused major complications at a rate of $3 \%$. These major complications included ST segment elevation on electrocardiography, troponin elevations, atrial fibrillation, and ventricular tachycardia. Readdy et al. ${ }^{27}$ used dopamine as the first-line vasopressor in $79 \%$ of patients and phenylephrine as the first-line agent in $21 \%$ of patients. Thirty percent of patients receiving dopamine experienced atrial fibrillation, ventricular tachycardia, or elevated troponins. Only $4.5 \%$ of patients receiving phenylephrine experienced these complications.

Dopamine and phenylephrine have historically been the primary vasopressors used in the study of SCI. Epinephrine and dobutamine have generally been avoided due to their cardiac side effects. ${ }^{17}$ Norepinephrine was used in multiple studies and historically has been a second- or third-line agent. In the study by Vale et al. ${ }^{31}$ norepinephrine was used as a second-line vasopressor to supplement dopamine. Hawryluk et al. ${ }^{16}$ only used norepinephrine in $1 \%$ of their patients as a first-line vasopressor. Inoue et al. ${ }^{17}$ administered norepinephrine to $5 \%$ of their patients, and Dakson et al. ${ }^{11}$ used norepinephrine exclusively in their study. There has been increasing interest and use of norepinephrine as the vasopressor of choice for SCI due to the demonstrated higher complication rate associated with dopamine use in SCI patients. There is also increasing evidence in the intensive care unit literature showing dopamine is associated with a greater mortality rate as well as being more arrhythmogenic compared with other vasopressors. ${ }^{12,13}$

There are limited data available for adequate comparison of phenylephrine versus norepinephrine as first-line agents in mid- to low thoracic SCI, and the recommendations have largely been based on the pharmacological properties of these agents. ${ }^{10,14,18,26,29}$ The pure alpha agonist activity of phenylephrine results in a decreased risk of arrhythmogenic side effects, although reflex bradycardia and peripheral vasoconstriction can occur. Norepinephrine's mixed alpha and beta effect decreases the risk of bradycardia and vasoconstriction; however, its positive inotropic and chronotropic effects can result in arrhythmias and other cardiac complications.

\section{Discussion}

Although there has been significant interest in the topic of promoting hypertension in SCI patients in the acute postinjury phase for the past several decades, there is limited and low-quality evidence regarding the risks and benefits of this practice. The basis of MAP goals and duration are most commonly attributed to 2 articles from the 1990s, which were both prospective studies reporting the goal of elevation of MAP for a specified duration of time postinjury. ${ }^{20,31}$ Based on a sound theoretical basis, other retrospective reviews and case series, and anecdotal reports, the practice of promoting elevated MAP goals is widely practiced and is formally recommended by the AANS/CNS Joint Committee guidelines. ${ }^{32}$

There are risks, however, associated with establishing elevated MAP in the period after SCI, which include complications due to vasopressor use, invasive monitoring, decreased patient mobilization, and prolonged hospitalization. Some of these risks, in particular vasopressor use, have been quantified and demonstrated to cause potentially major complications. Presently, the risk-benefit profile for vasopressor use is unclear, given the lack of definitive high-level evidence of BP augmentation in improving neurological recovery after SCI.

With regard to the optimal MAP, there have been no comparison studies to date, randomized or nonrandomized, comparing differences in outcome with different MAP goals. The formal recommendation of MAP of 85$90 \mathrm{~mm} \mathrm{Hg}$ appears to be derived from studies of Levi and Vale and their colleagues, ${ }^{20,31}$ in which MAP goals of 90 and $85 \mathrm{~mm} \mathrm{Hg}$ were chosen, respectively, without a clear explanation. There is the possibility that lower MAP goals may achieve similar results with less risk. Given that BP augmentation is currently the standard of care after SCI based on current recommendations, future studies in this patient population involving control groups will have to carefully consider the potential ethical questions of providing nonstandard-of-care treatment to a control group. This question is currently under investigation via the MAPS trial: Mean Arterial Pressure in Spinal Cord Injury (MAPS): Determination of Noninferiority of a Mean Arterial Pressure Goal of $65 \mathrm{~mm} \mathrm{Hg}$ Compared with a Mean Arterial Pressure Goal of $85 \mathrm{~mm} \mathrm{Hg}$ in Acute $\mathrm{Hu}-$ man Traumatic Spinal Cord Injury (clinicaltrials.gov no. NCT02232165). ${ }^{30}$

The duration of maintaining elevated MAP is currently recommended at 7 days, although no studies have compared different durations. A number of retrospective review papers have reported pursuing elevated MAP goals for a total of 5 days and did not indicate adverse outcomes related to this duration.

Recommended vasopressors for BP augmentation in SCI patients have historically generally been phenylephrine for mid- to low thoracic injuries and dopamine for high thoracic and cervical injuries, given its alpha and beta adrenergic effect. ${ }^{10}$ Norepinephrine has been used as well, but to a much lesser degree. Over the past several 
years, there has been increasing evidence that norepinephrine is superior to dopamine in the treatment of shock; the authors of a randomized controlled trial reported a higher mortality and complications with dopamine use. ${ }^{24}$ Although this trial was not conducted in SCI patients, the results are certainly worth consideration.

\section{Limitations}

This review is significantly limited by a number of important factors. At the individual study level, there were often low numbers of patients, follow-up was limited, and almost all of the studies lacked comparison groups. Across each study, there were different MAP goals and outcome measures, and protocols differed significantly. At the review level, there is the potential that our search did not uncover all relevant research, the possibility of reporting bias and publication bias, and the potential for not reporting all significant published information.

\section{Conclusions}

BP management in the acute period after SCI is a treatment of significant importance given the severe morbidity associated with SCI. Unfortunately, there is limited highquality evidence to guide BP management, and further research is essential. Based on the prospective studies, which provide the highest level of evidence available, MAP goals of $85-90 \mathrm{~mm} \mathrm{Hg}$ for 5-7 days postinjury should be considered. With regard to the optimal vasopressor, dopamine should be avoided. Norepinephrine should be considered as a first-line agent for cervical and upper thoracic SCIs, given evidence that it has a lower risk profile than dopamine. For SCIs in the mid- to lower thoracic spine, norepinephrine or phenylephrine should be considered as firstline agents.

\section{References}

1. Ahuja CS, Martin AR, Fehlings M: Recent advances in managing a spinal cord injury secondary to trauma. F1000 Res $\mathbf{5 : 5 , 2 0 1 6}$

2. Ahuja CS, Nori S, Tetreault L, Wilson J, Kwon B, Harrop J, et al: Traumatic spinal cord injury-repair and regeneration. Neurosurgery 80(3S):S9-S22, 2017

3. Ahuja CS, Wilson JR, Nori S, Kotter MRN, Druschel C, Curt A, et al: Traumatic spinal cord injury. Nat Rev Dis Primers 3:17018, 2017

4. Benzel EC, Larson SJ: Functional recovery after decompressive spine operation for cervical spine fractures. Neurosurgery 20:742-746, 1987

5. Bourassa-Moreau É, Mac-Thiong JM, Ehrmann Feldman D, Thompson C, Parent S: Complications in acute phase hospitalization of traumatic spinal cord injury: does surgical timing matter? J Trauma Acute Care Surg 74:849-854, 2013

6. Catapano JS, John Hawryluk GW, Whetstone W, Saigal R, Ferguson A, Talbott J, et al: Higher mean arterial pressure values correlate with neurologic improvement in patients with initially complete spinal cord injuries. World Neurosurg 96:72-79, 2016

7. Chehrazi B, Wagner FC Jr, Collins WF Jr, Freeman DH Jr: A scale for evaluation of spinal cord injury. $\mathbf{J}$ Neurosurg 54:310-315, 1981

8. Chesnut RM, Marshall SB, Piek J, Blunt BA, Klauber MR, Marshall LF: Early and late systemic hypotension as a frequent and fundamental source of cerebral ischemia following severe brain injury in the Traumatic Coma Data Bank. Acta Neurochir Suppl (Wien) 59:121-125, 1993

9. Cohn J, Wright J, McKenna S, Bushnik T: Impact of mean arterial blood pressure during the first seven days post spinal cord injury. Top Spinal Cord Inj Rehabil 15:96-106, 2010

10. Consortium for Spinal Cord Medicine: Early acute management in adults with spinal cord injury: a clinical practice guideline for health-care professionals. J Spinal Cord Med 31:403-479, 2008

11. Dakson A, Brandman D, Thibault-Halman G, Christie SD: Optimization of the mean arterial pressure and timing of surgical decompression in traumatic spinal cord injury: a retrospective study. Spinal Cord [epub ahead of print], 2017

12. De Backer D, Aldecoa C, Njimi H, Vincent JL: Dopamine versus norepinephrine in the treatment of septic shock: a meta-analysis. Crit Care Med 40:725-730, 2012

13. De Backer D, Biston P, Devriendt J, Madl C, Chochrad D, Aldecoa C, et al: Comparison of dopamine and norepinephrine in the treatment of shock. N Engl J Med 362:779-789, 2010

14. Ellender TJ, Skinner JC: The use of vasopressors and inotropes in the emergency medical treatment of shock. Emerg Med Clin North Am 26:759-786, ix, 2008

15. Haller J, Bice M, Lawrence B: Mediating the secondary effects of spinal cord injury through optimization of key physiologic parameters. J Am Acad Orthop Surg 24:160-171, 2016

16. Hawryluk G, Whetstone W, Saigal R, Ferguson A, Talbott J, Bresnahan J, et al: Mean arterial blood pressure correlates with neurological recovery after human spinal cord injury: analysis of high frequency physiologic data. J Neurotrauma 32:1958-1967, 2015

17. Inoue T, Manley GT, Patel N, Whetstone WD: Medical and surgical management after spinal cord injury: vasopressor usage, early surgerys, and complications. J Neurotrauma 31:284-291, 2014

18. Jia X, Kowalski RG, Sciubba DM, Geocadin RG: Critical care of traumatic spinal cord injury. J Intensive Care Med 28:12-23, 2013

19. Kepler CK, Schroeder GD, Martin ND, Vaccaro AR, Cohen M, Weinstein MS: The effect of preexisting hypertension on early neurologic results of patients with an acute spinal cord injury. Spinal Cord 53:763-766, 2015

20. Levi L, Wolf A, Belzberg H: Hemodynamic parameters in patients with acute cervical cord trauma: description, intervention, and prediction of outcome. Neurosurgery 33:10071017, 1993

21. Martin ND, Kepler C, Zubair M, Sayadipour A, Cohen M, Weinstein M: Increased mean arterial pressure goals after spinal cord injury and functional outcome. J Emerg Trauma Shock 8:94-98, 2015

22. Moher D, Liberati A, Tetzlaff J, Altman DG: Preferred reporting items for systematic reviews and meta-analyses: the PRISMA statement. Ann Intern Med 151:264-269, W264, 2009

23. National Spinal Cord Injury Statistical Center: Facts and Figures at a Glance. Birmingham: University of Alabama at Birmingham, 2016 (https://www.nscisc.uab.edu/Public/ Facts\%202016.pdf) [Accessed September 15, 2017]

24. Patel GP, Grahe JS, Sperry M, Singla S, Elpern E, Lateef O, et al: Efficacy and safety of dopamine versus norepinephrine in the management of septic shock. Shock 33:375-380, 2010

25. Piepmeier JM, Lehmann KB, Lane JG: Cardiovascular instability following acute cervical spinal cord trauma. Cent Nerv Syst Trauma 2:153-160, 1985

26. Ploumis A, Yadlapalli N, Fehlings MG, Kwon BK, Vaccaro AR: A systematic review of the evidence supporting a role for vasopressor support in acute SCI. Spinal Cord 48:356362,2010

27. Readdy WJ, Whetstone WD, Ferguson AR, Talbott JF, Inoue 
$\mathrm{T}$, Saigal R, et al: Complications and outcomes of vasopressor usage in acute traumatic central cord syndrome. J Neurosurg Spine 23:574-580, 2015

28. Simard JM, Tsymbalyuk O, Keledjian K, Ivanov A, Ivanova S, Gerzanich V: Comparative effects of glibenclamide and riluzole in a rat model of severe cervical spinal cord injury. Exp Neurol 233:566-574, 2012

29. Stevens RD, Bhardwaj A, Kirsch JR, Mirski MA: Critical care and perioperative management in traumatic spinal cord injury. J Neurosurg Anesthesiol 15:215-229, 2003

30. Tee JW, Altaf F, Belanger L, Ailon T, Street J, Paquette S, et al: Mean arterial blood pressure management of acute traumatic spinal cord injured patients during the pre-hospital and early admission period. J Neurotrauma 34:1271-1277, 2017

31. Vale FL, Burns J, Jackson AB, Hadley MN: Combined medical and surgical treatment after acute spinal cord injury: results of a prospective pilot study to assess the merits of aggressive medical resuscitation and blood pressure management. J Neurosurg 87:239-246, 1997

32. Walters BC, Hadley MN, Hurlbert RJ, Aarabi B, Dhall SS, Gelb DE, et al: Guidelines for the management of acute cervical spine and spinal cord injuries: 2013 update. Neurosurgery 60 (Suppl 1):82-91, 2013

33. Wolf A, Levi L, Mirvis S, Ragheb J, Huhn S, Rigamonti D, et al: Operative management of bilateral facet dislocation. $\mathbf{J}$ Neurosurg 75:883-890, 1991

34. Yashon D, Bingham WG Jr, Faddoul EM, Hunt WE: Edema of the spinal cord following experimental impact trauma. J Neurosurg 38:693-697, 1973

\section{Disclosures}

Dr. Park reports the following: consultant for Globus, Medtronic, NuVasive, and Zimmer Biomet; and royalties from Globus.

\section{Author Contributions}

Conception and design: Park, Smith. Acquisition of data: Saadeh, Jaffer. Analysis and interpretation of data: Park, Saadeh. Drafting the article: Park, Saadeh. Critically revising the article: Park, Saadeh, Smith, Joseph. Reviewed submitted version of manuscript: Park, Saadeh, Smith, Joseph, Buckingham, Oppenlander, Szerlip. Approved the final version of the manuscript on behalf of all authors: Park. Administrative/technical/material support: Park, Saadeh, Jaffer. Study supervision: Park, Smith, Joseph, Buckingham, Oppenlander, Szerlip.

\section{Correspondence}

Paul Park, Department of Neurosurgery, University of Michigan, 1500 East Medical Center Dr., Taubman Center, Rm. 3552, Ann Arbor, MI 48109. email: ppark@med.umich.edu. 